LIAMES 7 - pp. 41-60, Primavera 2007

Marisa Censabella

(Núcleo de Estudios en Lenguas Minoritarias Americanas (NELMA)

Instituto de Investigaciones Geohistóricas - CONICET Universidad

Nacional del Nordeste, Argentina)

\title{
Criterios de asignación de género gramatical en toba
}

\begin{abstract}
'Genders' or 'noun classes' are noun categorization devices that obligatory need agreement between 'controller' and 'target' classes (Corbett, 1991; Aikhenvald, 2000). In Toba (Guaykuruan), grammatical and natural gender are expressed in the opposition 'masculine / feminine' observed in the target classes (demonstrative dependents and pronominal demonstrative dependents) with non-derivate nouns, and also in the controller morpheme when it is constructed by derivation. In this paper we present the expression of gender (in target and controller classes), and the semantic, phonological and morphological rules of gender assignment in Toba. Semantic and phonological assignment rules are clearly identifiable but morphological assignment rule are more complex. Each derivational suffix selects always the same gender with one exception, the suffix $-k i$. This suffix that could be interpreted as nominal classifier controls masculine or feminine gender on the basis of semantic criteria, as shape or volume. Also related to the morphological assignment rules, we have identified processes of reanalysis where derivational suffixes associated with shape information are reinterpreted as gender markers. Finally, we present the gender assignment in Spanish loans and in cases of homophony.

KEYWORDS: Noun categorization; Gender; Classifiers; Toba; Guaycuruan.
\end{abstract}

\section{RESUMEN}

El género es uno de los tipos de categorización nominal que se diferencia de otros -los clasificadores nominales, por ejemplo- por exigir concordancia entre una clase 'controladora' y la/s clase/s 'meta' (Corbett, 1991; Aikhenvald, 2000). En toba (familia Guaycurú), la marcación de género natural y gramatical se organiza en la oposición masculino / femenino, y se manifiesta por medio de afijos en los dependientes demostrativos o en los mismos nombres, cuando estos son derivados. En este trabajo presentamos las clases de palabras que expresan el género y sus reglas de asignación semántica, fonológica y morfológica. Las reglas de asignación semántica y fonológica no presentan problemas para su identificación. En cuanto a la asignación morfológica, cada sufijo nominalizador selecciona la misma marca de género gramatical, salvo los nombres derivados que utilizan el sufijo - $k i$ 'lugar cerrado, posición vertical', caso en que puede seleccionar tanto uno como otro género; además se observa procesos de 
nombres, cuando estos son derivados. En este trabajo presentamos las clases de palabras que expresan el género y sus reglas de asignación semántica, fonológica y morfológica. Las reglas de asignación semántica y fonológica no presentan problemas para su identificación. En cuanto a la asignación morfológica, cada sufijo nominalizador selecciona la misma marca de género gramatical, salvo los nombres derivados que utilizan el sufijo - $k i$ 'lugar cerrado, posición vertical', caso en que puede seleccionar tanto uno como otro género; además se observa procesos de reanálisis de sufijos indicadores de forma en marcadores de género natural. Finalmente, se presenta la expresión del género en préstamos y su comportamiento en casos de homofonía.

PALABRAS CLAVE: Categorización nominal; Género; Clasificadores, Toba; Familia Guaycurú.

\section{INTRODUCCIÓN}

La tipología funcional presta especial atención al estudio de los clasificadores y de los sistemas de categorización nominal porque brindan una vía de acceso único para investigar la manera en que los seres humanos categorizan el mundo a través del lenguaje (Aikhenvald, 2000: 2-5). Siguiendo la clasificación de esta autora, la lengua toba cuenta con cuatro tipos de morfemas que indican categorización nominal: las clases nominales o 'géneros', los clasificadores deícticos, los clasificadores posesivos y los clasificadores nominales. Los clasificadores deícticos han sido los más estudiados, tanto para el toba como para otras lenguas guaycurúes: Klein (1979), Buckwalter (1980), Messineo (1998) y Censabella (2001) para el toba; Kirtchuk (1996) y Vidal (1997) para el pilagá; Sándalo (1995) para el caduveo; y Gualdieri (1998, 2007) y Grondona (1998) para el mocoví. Menos Kirtchuk, todos los autores mencionados presentan los clasificadores nominales -identificados como tales o bien como sufijos nominalizadores-, mientras que Messineo (2002) estudió los clasificadores posesivos en toba.

En este trabajo presentamos los criterios semánticos, fonológicos y morfológicos de asignación de género en la lengua toba y proponemos explicaciones a los fenómenos de reanálisis observados. Para la realización de este trabajo se utilizó la información sobre el género gramatical de cada ítem léxico del Vocabulario Toba de Alberto Buckwalter (1980) y un corpus propio de diez horas de texto libre narrativo.

\section{EXPRESIÓN MORFOLÓGICA DEL GÉNERO NATURAL Y GRAMATICAL}

Es bien conocida la diferencia que realizan muchos lingüistas entre sexo y género (Martinet, 1985: 55), o entre género natural y género gramatical (Trudgill, 2002: 76). En el primer caso se trata de distinciones realizadas sobre una base semántica, utilizadas para marcar la diferencia sexual entre seres humanos y mamíferos superiores. En el segundo se trata de una extensión metafórica de la noción de género natural (Trudgill, 2002: 77) pero que no responde 'icónicamente' a distinciones sexuales, dado que sus referentes son objetos inanimados o nociones abstractas. Hablamos de género natural, entonces, cuando el hablante está obligado a indicar el sexo de un ser humano o ser animado y elije, según el referente, el 'masculino' o el 'femenino': hermano/hermana, hijo/hija, por extensión para algunos animales domésticos: perro / perra ${ }^{1}$. Por el contrario, si el hablante no puede elegir 
diferencia sexual entre seres humanos y mamíferos superiores. En el segundo se trata de una extensión metafórica de la noción de género natural (Trudgill, 2002: 77) pero que no responde 'icónicamente' a distinciones sexuales, dado que sus referentes son objetos inanimados o nociones abstractas. Hablamos de género natural, entonces, cuando el hablante está obligado a indicar el sexo de un ser humano o ser animado y elije, según el referente, el 'masculino' o el 'femenino': hermano / hermana, hijo / hija, por extensión para algunos animales domésticos: perro / perra ${ }^{1}$. Por el contrario, si el hablante no puede elegir el género de un nombre, estamos frente a un caso de género gramatical: debe decir el lápiz y no *la lápiz, tal como se lo indica el lexicón de su lengua.

Para Corbett (1991: 4-5), la concordancia reflejada en las clases de palabras asociadas a los nombres permite identificar el número de 'géneros' que posee una lengua, que puede sobrepasar la distinción sexual entre masculino y femenino e incluir otros clasificadores nominales (animados / inanimados, animales domésticos / animales salvajes, clasificadores de forma, etc. $)^{2}$. De esta forma Corbett diferencia 'géneros' de 'clasificadores' ('class classifiers' o 'nominal classifiers') ya que estos últimos no desencadenan fenómenos de concordancia, aun cuando puedan ser el origen de los sistemas de género.

\section{LA LENGUA TOBA}

La lengua toba pertenece a la familia lingüística Guaycurú, junto al pilagá, mocoví, caduveo y a las ya extintas abipón y mbayá. Estimamos que es hablada -con diversos grados de competencia, tanto en ámbitos rurales como urbanos- por más de la mitad de las 69.452 personas de origen toba ${ }^{3}$ que habitan en las provincias de Chaco, Formosa y Salta y, debido a movimientos migratorios, en las ciudades de Rosario (Santa Fe) y periferia de Buenos Aires, en la República Argentina. En la localidad de El Cerrito, a 60 Km. al NO de Asunción (Paraguay) se encuentra alrededor de 500 hablantes de la variedad denominada emok-toba (Messineo, 1991). Se trata de una lengua en peligro, ya que es progresivamente desplazada por el español, especialmente en las zonas urbanas.

Con tendencia a la polisíntesis, orden VS, AVO y OVA cuando O es pronominal, la lengua toba posee oposición verbo/nombre, sistema pronominal subjetivo incorporado al verbo, no posee adjetivos como clase de palabras ni marca caso. Distingue nombres alienables de inalienables. Posee cuatro tipos de morfemas categorizadores nominales: classes nominales ('masculino' y 'femenino'), classificadores nominales (de forma),

\footnotetext{
${ }^{1}$ En la mayoría de las lenguas, las distinciones de género natural en animales se indican mediante procedimentos supletivos, en este caso léxicos: toro/ vaca, caballo/ yegua, etc.

${ }^{2}$ Un ejemplo bastante conocido en la literatura lingüística es la clasificación genérica de la lengua Dyirbal, hablada al NE de Queensland (Australia), cuyos nombres se clasifican en cuatro géneros: I (bayi): hombres y animados no-humanos; II (balan): mujeres, agua, fuego, lucha; III (balam): miel y plantas y vegetales comestibles; IV (bala): el resto de las categorias (partes del cuerpo, viento, los árboles, las piedras, el lenguaje, etc). (Corbett, 1991: 15-16, citando a Dixon, R. M. W. 1972. The Dyirbal Language of North Queensland. Cambridge University Press).

${ }^{3}$ Datos tomados de la Encuesta Complementaria de Pueblos Indígenas realizada por el Instituto Nacional de Estadísticas y Censos en los años 2004 y 2005 (Indec, 2007).
} 
clasificadores posesivos y clasificadores deícticos. El número se expresa a través de la oposición singular, paucal y plural; posee marca morfológica de distributivo y de colectivo, estas dos últimas como clases de morfemas diferentes del número propiamente dicho, y expresión sintáctica del número dual.

Los verbos muestran dos paradigmas de conjugación para todas las personas, el de voz activa y el de voz media. En la voz activa, y sólo para la tercera persona singular y plural, se observan rasgos de ergatividad al utilizar marcas diferentes para $\mathrm{A}$ y $\mathrm{S}$ en los personales verbales. A su vez, la expresión de $\mathrm{S}$ en la tercera persona activa está escindida en tres formas, las cuales se encuentran en su gran mayoría lexicalizadas, e indican distintos grados de control y afectación del participante único. Para un puñado de raíces verbales subsiste una marcación del tipo activa/inactiva, observable en la 1ra persona del singular y del plural. La lengua no marca tiempo verbal y posee un sistema aspectual organizado en la oposición perfectivo/imperfectivo y otros modos de acción. Cuenta con dos paradigmas de adposiciones verbales, uno de direccionales y otro de locativo-orientativos, estos últimos se comportan como aplicativos. Las operaciones de modificación de la valencia son reflexivo, recíproco, antipasiva, seis construcciones causativas morfológicas, pasiva no-promocional y varias construcciones aplicativas. La lengua permite relativizar sujetos y objetos, y posee un patrón de coordinación de cláusulas nominativo-acusativo (pivote S/A). (Censabella, 2002, 2006; Carpio, 2004).

\section{LAEXPRESIÓNDEL GÉNEROENTOBA}

En toba, las clases de palabras portadoras de la noción de género son los nombres alienables e inalienables (simples y derivados), el pronombre personal de 3ra persona, los pronombres demostrativos y los nombres propios ${ }^{4}$. En nombres alienables e inalienables, el género se manifiesta en los dependientes o 'clases meta', y en ciertos nombres derivados, especialmente si indican género natural, éste se expresa también en el núcleo. La expresión del género se verifica en todos los argumentos de la cláusula sin importar su función, sea ésta $\mathrm{S}, \mathrm{A}, \mathrm{O}$ u Oblicuo.

\subsection{Identificación}

La asignación de género gramatical se indica mediante la concordancia entre una clase 'controladora'-en nuestro caso los nombres alienables e inalienables- y la clase 'meta' (Corbett, 1991: 150) que en toba es el determinante demostrativo o el pronombre demostrativo. En el ejemplo (1), se comprueba que los controladores waloq laji 'trapo de algodón' y 'naraanxa' 'naranja' son masculino y femenino respectivamente, ya que la clase 'meta' pronombre demostrativo, concuerda con ellos:

\footnotetext{
${ }^{4}$ En este trabajo no presentamos la asignación de género en nombre propios. Aunque contamos con la lista de nombres masculinos y femeninos presentada por Buckwalter (1980): 530), nos faltam nombres propios tobas insertos en cláusulas donde observar los fenómenos de concordancia. Como es de esperar, en los nombres propios prima el criterio de asignación de género semántico, aun cuando viole las reglas de asignación fonológica (ver punto 3.4.2).
} 
(1)

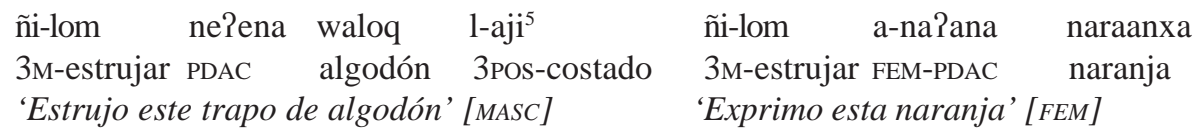

En los ejemplos (2) las clases meta son los determinantes demostrativos -no pronominales- na y ana que permiten identificar el género de los nombres inalienables $i$ wel 'mi vientre' y l-ere 'su libro' como masculino y femenino respectivamente:

(2)

$\begin{array}{llllll}\text { so-wet } & \text { na } & \text { i-wel } & \text { i-men } & \text { a-na } & \text { l-ere } \\ \text { 1-doler } & \text { DAC } & \text { 1POS-vientre } & \text { 3T-comprar } & \text { FEM-DAC } & \text { 3POS-libro, cuaderno } \\ \text { 'Me duele este mi vientre' [MASC] } & \text { 'Compra este su libro' [FEM] } & \end{array}$

En los textos libres se observa que la mayoría de los nombres alienables e inalienables son masculinos, en uno de ellos - de cien enunciados aproximadamente- se utilizaron 212 nombres masculinos y sólo 29 femeninos $^{6}$. La información referida al género en el voluminoso Vocabulario Toba de Alberto Buckwalter (1980) se presenta de manera poco sistemática: los nombres femeninos van acompañados de sus dependientes, como en (3) donde se deduce que ambos lexemas son femeninos gracias a la expresión del género en el dependiente $a \tilde{n} i^{7}$ :

$\begin{array}{ll}\text { (3) } & \text { 'sajte, añi: } \\ \text { waGayaGa, añi: } & \text { 'el zorro' }\end{array}$

pero para los lexemas masculinos el criterio de clasificación es opaco; en algunos casos la entrada léxica va acompañada de una oración que permite identificar el género:

$$
\text { lajta 'su olor' ama na Rajta 'tenés lindo olor' }
$$

de lo que se deduce que laita 'su olor' es masculino porque el dependiente es na y no ana. En otros casos, al no presentar cláusulas, es imposible saber si la falta de asignación de género indica que el nombre es masculino, o bien que el autor no pudo relevar esa información, como en (5) donde el lexema kote 'palometa', de género femenino según nuestro análisis (ver más abajo 3.4.2), no está acompañado del dependiente añi como en los ejemplos (3):

\footnotetext{
${ }^{5}$ Utilizamos la transcripción del APHI salvo ñ, ch y sh que corresponden a los simbolos $\mathrm{n}, \mathrm{t} \int \mathrm{y} \int$ respectivamente.

${ }^{6}$ Si bien la elicitación de las marcas de género es la metodología de relevamiento recomendada por Aikhenvald (2000: 447), no resulta sencillo hacerlo con hablantes bilingües toba-español urbanizados y escolarizados. En muchos casos hemos detectado dudas acerca de la asignación de género y calcos del español. Aunque siempre es possible trabajar con hablantes ancianos, cuya competencia está más libre de hipercorrecciones, consideramos que los textos libres son la fuente más confiable para analisar los criterios de asignación de género.

Transcribimos los ejemplos de Buckwalter con la notación del APHI.
} 
(5) kote 'la palometa'

\subsection{Nombres alienables e inalienables}

3.2.1. Género gramatical

En los nombres alienables (6) e inalienables (7), la codificación del género gramatical se manifiesta en los dependientes demostrativos. En estos casos, el valor marcado es el femenino:

\begin{tabular}{|l|l|l|}
\hline$\varnothing-$ & masculino & MARCADO EN EL \\
\hline a- & femenino & DEPENDIENTE \\
\hline
\end{tabular}

Tabla 1. Género gramatical en nombres alienables e inalienables.

(6)

$\begin{array}{llll}\text { na } & \text { koki } & \text { a-na } & \text { lachi } \\ \text { DAC } & \text { plato } & \text { FEM-DAC } & \text { cebolla } \\ & \text { 'este plato' [MASC] } & \begin{array}{l}\text { 'esta cebolla' } \\ \text { [FEM] }\end{array} \\ \text { na } & \text { 1-qajk } & \text { a-na } & \text { 1-asoge? } \\ \text { DAC } & \text { 3POS-cabeza } & \text { FEM-DAC } & \text { 3POS-bolsa } \\ \text { 'esta su cabeza' [MASC] } & \text { 'esta su bolsa' } & \text { [FEM] }\end{array}$

\subsubsection{Género natural}

El género natural en nombres alienables e inalienables derivados se manifiesta tanto en el dependiente demostrativo como en el núcleo. En el núcleo la marcación de género es inversa a la vista en los dependientes: no-marcado para el femenino y marcado para el masculino, mientras que la clase 'meta' presenta el tipo de marcación visto en 3.2.1:

\begin{tabular}{|l|l|l|}
\hline$-\mathrm{k}$ & masculino & MARCADO EN EL \\
\hline$-\varnothing$ & femenino & NÚCLEO \\
\hline
\end{tabular}

Tabla 2. Género natural expresado en nombres alienables e inalienables derivados.

$$
\begin{array}{lll}
\text { (8) } \quad \begin{array}{ll}
\text { ra } \\
\text { DPA }
\end{array} & \begin{array}{l}
\text { 1-jale-k } \\
\text { 3POS-hijo-MASC } \\
\text { 'este su hijo' }
\end{array} \\
& \text { ra } & \begin{array}{l}
\text { noGot-ole-k } \\
\text { joven-DIM-MASC } \\
\text { 'este niño' }
\end{array}
\end{array}
$$$$
\text { a-ra } \quad \text {-jale- } \varnothing
$$$$
\text { FEM-DPA 3POS-hijo-FEM }
$$$$
\text { 'esta su hija' }
$$

$\begin{array}{ll}\text { a-ra } & \text { noGot-ole- } \varnothing \\ \text { FEM-DPA } & \text { joven-DIM-FEM } \\ \text { 'esta niña' } & \end{array}$


Censabella: CRitérios de asignación de GÉNero gramatical EN TOBA

(10)

$\begin{array}{llll}\text { ra } & \text { choGon-aGa-j-k } & \text { a-ra } & \text { choGon-aGa-j- } \varnothing \\ \text { DPA } & \text { soñar-N1-P1-MASC } & \text { FEM-DPA } & \text { soñar-N1-P1-FEM } \\ \text { 'este soñador' } & \text { 'esta soñadora' } & \end{array}$

Esta lengua no cuenta con estudios comparativos y diacrónicos que nos permitan rastrear el origen léxica de los afijos de género. En otros subsistemas gramaticales de la lengua, el segmento $[. . . k]$ se utiliza para distinguir aspecto verbal continuo - $t a$ de aspecto verbal progresivo -tak, en cláusulas de este tipo: Juan ralolata 'Juan está enfermo' / Juan rasottak 'Juan está bailando'. Análogamente, un segmento similar se utiliza en los nombres de acciones para indicar aquellas realizadas con mayor volición y control, en contraste con aquellas donde dichos rasgos semánticos están ausentes: nalolaGa 'enfermedad' / nasotaGak 'baile'. Este podría ser un posible origen de la marcación genérica masculina $k$, la expresión de mayor agentividad, hipótesis que tendrá que ser corroborada en relación a las concepciones culturales tobas.

\subsection{Pronombres personales y demostrativos}

Los pronombres personales de tercera persona singular y plural expresan el género natural (11) al igual que los pronombres demostrativos, tanto en función deíctica (12) como anafórica (13). En ambos casos, el femenino es el caso marcado:

\begin{tabular}{|l|l|l|}
\hline$\varnothing-$ & masculino & $\begin{array}{l}\text { marcado en el núcleo cuando } \\
\text { cumplen función pronominal }\end{array}$ \\
\hline a- & femenino & \\
\hline
\end{tabular}

Tabla 3. Género natural expresado en pronombres personales y demostrativos.

(11)

$\begin{array}{ll}\text { ra-maze } & \text { r-kePe-tak } \\ \text { DPA-P3 } & \text { 3IA-comer-PROG }\end{array}$

a-ra-maze r-ke?e-tak

'él está comiendo'

FEM-DPA-P3 3IA-comer-PROG

$\begin{array}{llll}\text { ra-Pa-maze } & \text { r-aso?t } & \text { a-ra-Pa-maze } & \text { r-aso?t } \\ \text { DPA-PL-P3 } & \text { 3IA-bailar/pl } & \text { FEM-DPA-PL-P3 } & \text { 3IA-bailar/PL } \\ \text { 'ellos bailan' } & & \text { 'ellas bailan' } & \end{array}$

(12) ñi?iñi jale a-ñi?añi Palo

PDSE hombre FEM-PDSE mujer

'este hombre sentado' 'esta mujer sentada'

(13) ...qa-j-asaG-an-gi a-na lachewge ze?eze...

ADES-3T-arrojar-CAUS-APL:AD FEM-DAC río PDACOS

'... empujan adentro del río a aquel...' 


$\begin{array}{llll}\ldots \text { qqa-j-asaG-an-gi } & \text { a-na } & \text { lachewge } & \text { a-zePaze... } \\ \text { ADES-3T-arrojar-CAUS-APL:AD } & \text { FEM-DAC } & \text { río } & \text { FEM-PDACOS } \\ \text { ‘... empujan adentro del río a aquella....' } & & \end{array}$

\subsection{Criterios de asignación de género}

Un primer paso para identificar las reglas de asignación de género es dilucidar si se basan en criterios semánticos, morfológicos, fonológicos o en una combinación de ellos. Según Corbett (1991: 8), ningún sistema de asignación de género deja de poseer un fundamento semántico, ya que -inclusive en los casos en los que la selección está regida por razones morfológicas o fonológicas- por analogía o para evitar ambigüedades, primará el criterio semántico. La lengua toba utiliza los tres criterios de asignación del género: el semántico, el fonológico y el morfológico. Los presentaremos en ese orden para luego observar sus interrelaciones.

\subsubsection{Criterio semántico}

Para Corbett (1991: 8), el criterio de asignación semántica de género es aquel mediante el cual el significado de un nombre determina su género; es decir, dado el género de un nombre se puede inferir algo acerca de su significado. Operará este criterio en relación al sexo de seres humanos y otros seres vivos:

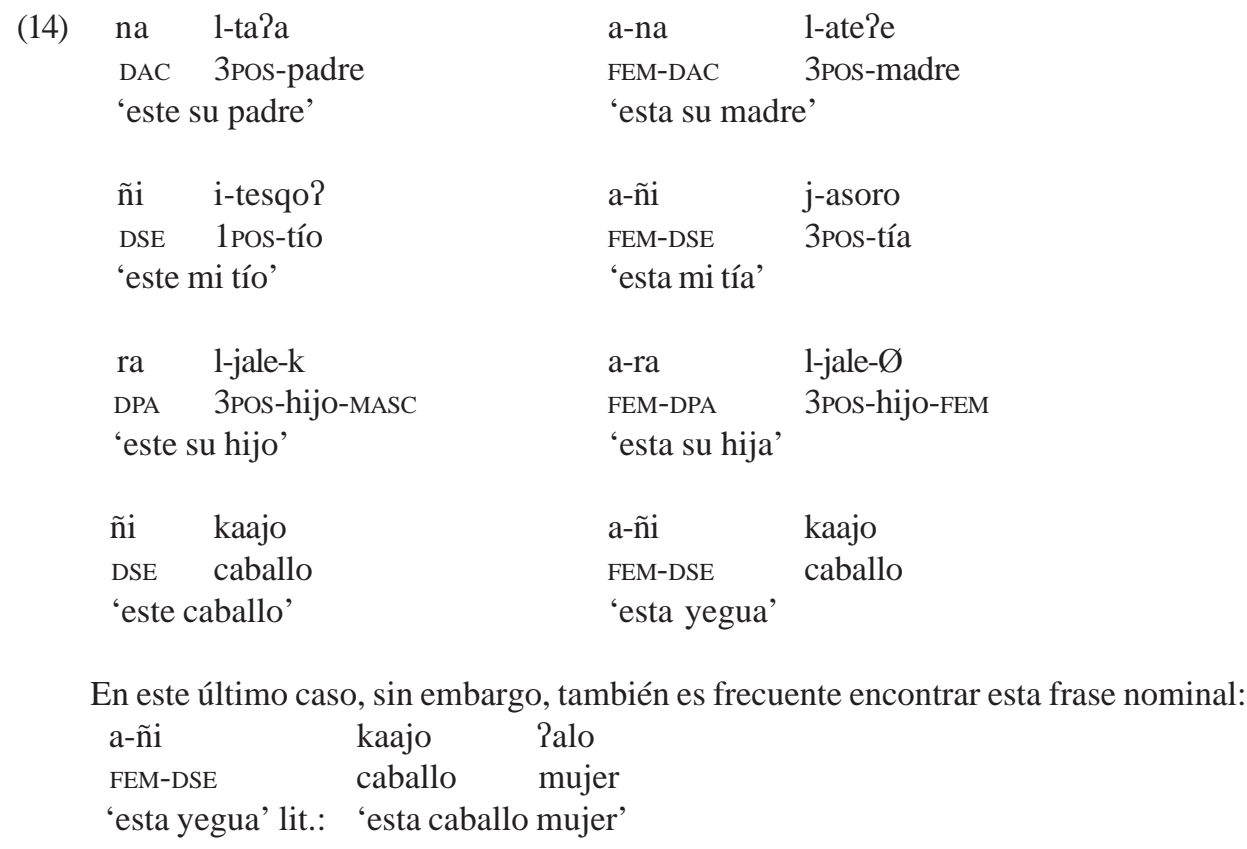


En lo que respecta a la clasificación del mundo vegetal, los tobas asignan género femenino a árboles, algunos cactus y variados frutos; la tierra -donde crecen las semillas- al igual que el sol-que hace crecer los frutos- serán femeninos, mientras que la luna, por el contrario, es masculina:

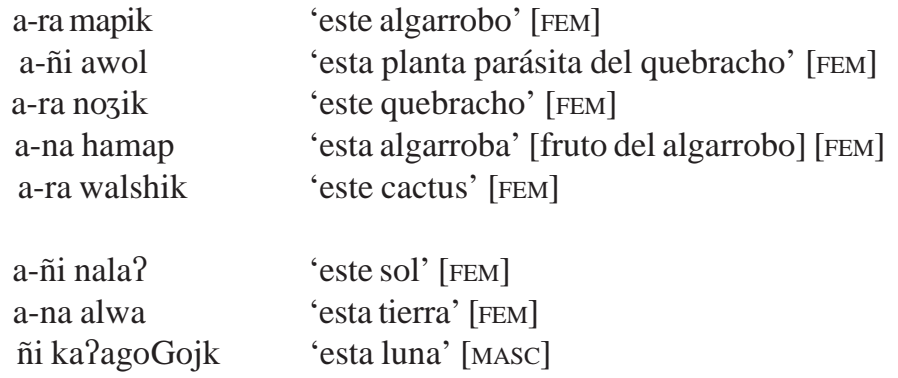

El género gramatical de animales y aves sigue un criterio semántico que responde a concepciones culturales tobas, como se puede verificar en el mito del Diluvio de fuego, en la versión relevada por Lehmann-Nitsche en 1924 y publicada en Wilbert \& Simoneau (1982: 25-26): un grupo de hombres y mujeres 'antiguos' se resguardan en una cueva mientras la tierra es asolada por un diluvio de fuego; cuando el fuego desaparece, estos personajes son alentados a salir de su escondite pero con la prohibición de mirar a su alrededor. Muchos de ellos no logran cumplir la orden y se transforman en animales: un hombre se convierte en iguana (na qolligesaq [MASC]), una mujer se transforma en oso hormiguero (ana potay [FEM]). Este criterio se verifica en totalidad de los animales citados en dicho mito y también en otras leyendas que tratan sobre transformaciones de humanos a animales. Otros nombres de animales son:

\begin{tabular}{|c|c|}
\hline $\begin{array}{l}\text { na qageta } \\
\text { 'esta oveja' [MASC] }\end{array}$ & $\begin{array}{l}\text { ana wa } \lambda \text { iqaGaj } \\
\text { 'este carpincho' [FEM] }\end{array}$ \\
\hline $\begin{array}{l}\text { na tonolek } \\
\text { 'este caburé' (lechuza) [MASC] }\end{array}$ & $\begin{array}{l}\text { añi taqaq } \\
\text { 'este chajá' [FEM] }\end{array}$ \\
\hline $\begin{array}{l}\text { na Pal } \\
\text { 'esta comadreja' [MASc] }\end{array}$ & $\begin{array}{l}\text { añi nchigishi } \\
\text { 'esta nutria' [FEM] }\end{array}$ \\
\hline $\begin{array}{l}\text { na cheGrañigo } \\
\text { 'este ciervo' [MASC] }\end{array}$ & $\begin{array}{l}\text { añi coshingo } \\
\text { 'este coatí' [FEM] }\end{array}$ \\
\hline
\end{tabular}

La expresión del género natural en nombres alienables e inalienables derivados referidos a humanos se expresa también en el núcleo: 
(17)

\begin{tabular}{|c|c|c|c|c|c|c|c|}
\hline na & 1-tela & na & tela- & & \multicolumn{2}{|c|}{ a-na } & ela-j-Ø \\
\hline DAC & 3POs-oreja & DAC & oreja & -P1-MASC & \multirow{2}{*}{\multicolumn{3}{|c|}{$\begin{array}{l}\text { FEM-DAC oreja-P1-FEM } \\
\text { 'esta orejuda' }\end{array}$}} \\
\hline \multicolumn{2}{|c|}{ 'esta su oreja' } & \multicolumn{3}{|c|}{ 'este orejudo' } & & & \\
\hline na & & na & lew-aG & & a-na & lew-aGa-y-Ø \\
\hline & \multirow{2}{*}{\multicolumn{2}{|c|}{$\begin{array}{l}\text { 3IPOS-morir-N1 } \\
\text { huerte' }\end{array}$}} & \multirow{2}{*}{\multicolumn{3}{|c|}{$\begin{array}{l}\text { DAC morir-N1-P1-MASC } \\
\text { 'este muerto' }\end{array}$}} & \multirow{2}{*}{\multicolumn{2}{|c|}{$\begin{array}{l}\text { FEM-DAC morir-N1-P1-FEM } \\
\text { 'esta muerta' }\end{array}$}} \\
\hline 'esta muerte' & & & & & & & \\
\hline na & ntap & & na & ntap-to- & & a-na & ntap-to- $\varnothing$ \\
\hline DAC & calor del sol & & DAC & c.d.s-p4 & & FEM-DAC & c.d.s-P4-FEM \\
\hline \multicolumn{2}{|c|}{ 'este calor del sol' } & & \multicolumn{3}{|c|}{ 'este insolado' } & 'esta ins & solada' \\
\hline
\end{tabular}

\subsubsection{Criterio fonológico}

Dentro de los criterios de asignación de género formales, es decir no semánticos, Corbett (1991: 51) establece el morfológico y el fonológico, ambos estrechamente emparentados según el autor. El criterio de asignación fonológico se comprueba cuando el género de un nombre puede ser identificado a partir de las propiedades formales del lexema, como puede ser un fonema final o alguna característica tonal o de acentuación. En toba, todos los nombres alienables e inalienables, simples o derivados, que finalizan en vocal - $e$ asignan género femenino. El carácter fonológico de este criterio de asignación se constata todavía más al comprobar que aquellos lexemas que finalizan con los segmentos -che; -chi; -she; -shi; -ze;-3i también asignan género femenino, porque la oposición entre las vocales e/i se neutraliza precedida de las fricativas prepalatales y la africada lámino-prepalatal (Censabella, 1997):

$\begin{array}{ll}\text { a-na lachi } & \text { 'este granizo, esta cebolla' [FEM] } \\ \text { a-na ware } & \text { 'este pedazo de cuero' [FEM] } \\ \text { a-na rawa?chi } & \text { 'este remolino de viento' [FEM] } \\ \text { a-na qaze } & \text { 'esta boleadora' [FEM] } \\ \text { a-na woqte } & \text { 'este horcón' [FEM] } \\ \text { a-ñi l-ere } & \text { 'este su libro' [FEM] } \\ \text { a-ñi l-we } & \text { 'este su diente' [FEM] } \\ \text { a-na l-ne } & \text { 'esta su espina finita' [FEM] } \\ \text { a-ñi l-malate } & \text { 'este su documento' [FEM] }\end{array}$

Este criterio fonológico opera cuando los nombres derivados mediante sufijos nominalizadores admiten el sufijo clasificador de forma 'objeto pequeño y/o redondo': 
(19)

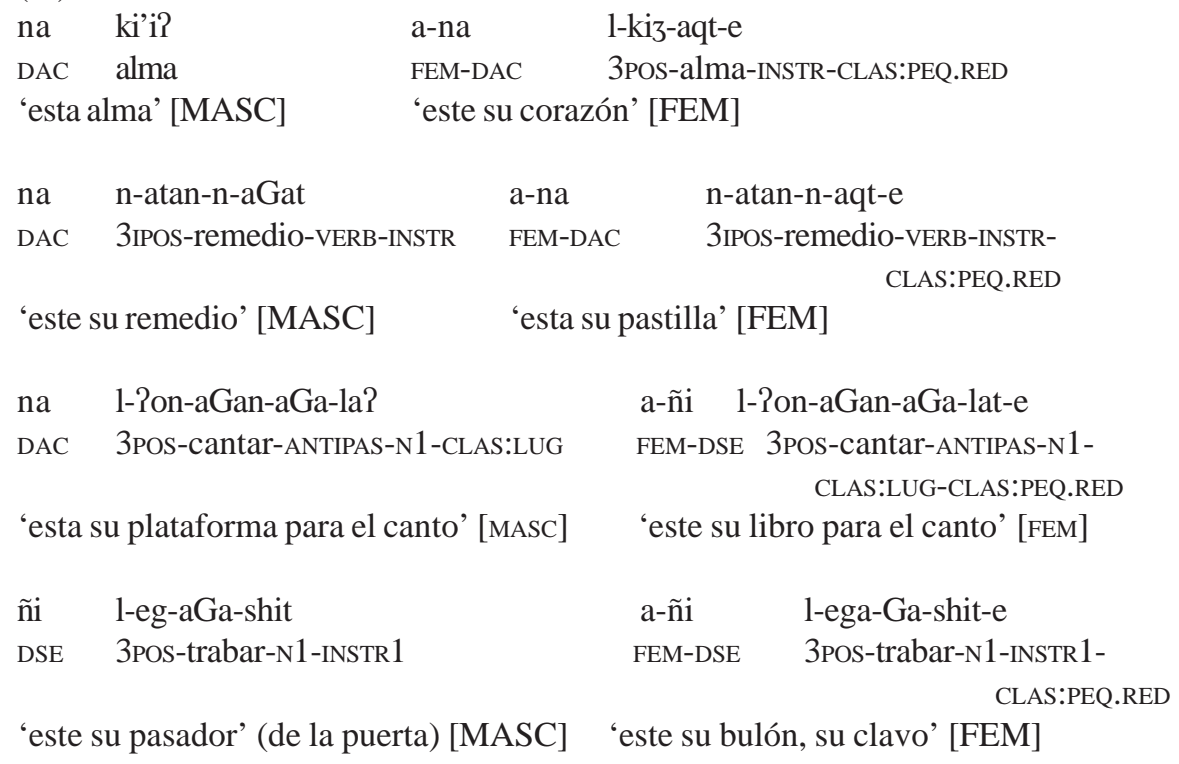

En los ejemplos (20) observamos una extensión metafórica del clasificador 'forma pequeña y/o redonda' en indicador de género natural, respetando igualmente la regla de asignación de género fonológica:

\begin{tabular}{|c|c|c|c|c|}
\hline qom & na & \multicolumn{2}{|c|}{ qom-1Rek-Ø } & qom-lash-e \\
\hline DAC gente toba & DAC & \multirow{2}{*}{\multicolumn{2}{|c|}{$\begin{array}{l}\text { toba-GENT-MASC } \\
\text { 'este hombre toba' }\end{array}$}} & FEM-DAC toba-GENT-CLAS:PEQ.RED \\
\hline \multicolumn{2}{|c|}{ 'esta gente', 'los tobas' } & & & 'esta mujer toba' \\
\hline na rokshe & \multirow{2}{*}{\multicolumn{2}{|c|}{$\begin{array}{l}\text { na rokshe-liek- } \varnothing \\
\text { DAC blanco-GENT-MASC }\end{array}$}} & a-na & rokshe-lash-e \\
\hline DAC gente blanca & & & \multicolumn{2}{|c|}{ FEM-DAC blanco-GENT- } \\
\hline & \multicolumn{2}{|c|}{ 'este hombre hlanco' } & & CLAS:PEQ.RED \\
\hline
\end{tabular}

\subsubsection{Criterio morfológico}

El otro criterio formal de asignación género, el morfológico, se comprueba cuando el género de un nombre puede ser identificado a partir de propiedades formales, como la presencia de un tipo de morfema derivativo o flexivo (Corbett, 1991: 34 y sig.). En toba, los nombres de acción de eventos pacientivos (21) y de eventos agentivos (22) y los morfemas nominalizadores 'LUGAR' (23), 'INSTRUMENTAL' (24) y 'CLASIFICADOR LOCATIVO' (25) seleccionan el criterio morfológico de asignación de género. En estos casos, tal como vimos en 3.2.1, el género se verifica en el dependiente, es decir en el determinante demostrativo: 
(21) i-lew

3T-morir

'(él/ella) muere'

(22) r-asot

3IA-bailar

'(él/ella) baila'

(23) i-men

3T-vender

'(él/ella) vende'

(24) y-ashi?

3T-soplar

'sopla'

(25) r-awoGon

3IA-asar

'asa' na n-lew-aGa

DAC 3IPOS-morir-N1

'esta su muerte' [MASC]

na n-asot-aGak

DAC 3IPOS-bailar-N2

'este su baile' [MASC]

na men-qa?

DAC vender-LUG

'este mercado (al aire libre)' [MASC]

na 1-asher-agat

DAC 3POS-soplar-INSTR

'este su ventilador' [MASC]

na $\quad$ l-awoGon-aGa-la?
DAC $\quad$ 3Pos-asar-N1-CLAs:AB.HORIZ
'esta su parrilla' [MASC]

Los nombres derivados (24) y (25) admiten el sufijo CLASIFICADOR:PEQUEÑO.REDONDO; en estos casos el nombre cambia al género femenino, siguiendo la regla fonológica presentada en 3.4.2:
a-na l-ashir-aqt-e
FEM-DAC 3POS-SOplar-INSTR-CLAS:PEQ.RED

'este su abanico' [FEM]
a-na l-arona-Ga-lat-e
FEM-DAC 3POS-casarse-N1-CLAS:AB.HORIZ-CLAS:PEQ.RED
‘su libreta de matrimonio' [FEM]

Los nombres derivados con sufijos 'nombre del objeto RESULTATIVO y PACIENTIVo de la acción verbal' también utilizan el criterio de asignación de género morfológico. La diferencia semántica entre estos dos tipos de derivados se observa claramente cuando los nombres derivan de verbos transitivos, el derivado resultativo selecciona el género masculino y el pacientivo el género femenino:

$\begin{array}{lll}\text { (28) } & \text { ya-qaleten } & \text { na l-qalehn-ek } \\ \text { 3T-hilar } & \text { DAC } 3 \text { POS-hilar-RES } \\ \text { '(ella) hila' } & \text { 'esta su manta hilada' [MASC] }\end{array}$


Censabella: Critérios de asignación de GÉnero gramatical en toba

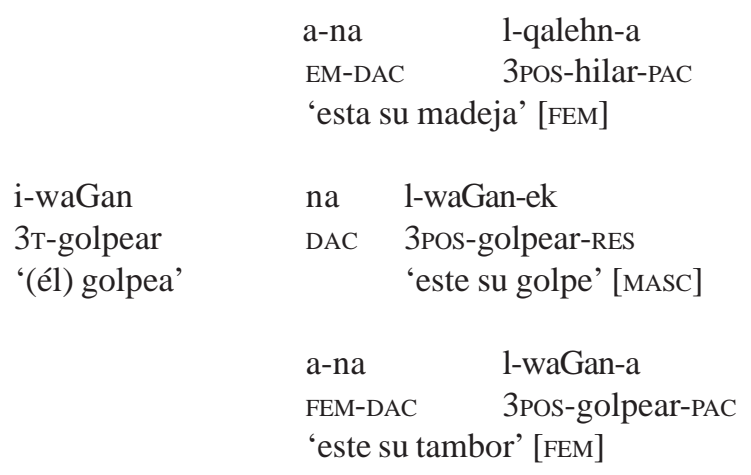

\subsection{Reanálisis de la asignación morfológica de género en nombres pacientivos y resultativos}

Hemos observado un proceso de reanálisis de la asignación de género en nombres derivados que utilizan los sufijos nominalizadores pacientivos y resultativos. Si éstos se derivan de bases verbales causativas intencionales, la diferencia entre ambos sufijos será reinterpretada como marca de género natural y los derivados serán, ambos, pacientivos: (29)

$\begin{array}{lll}\text { i-wosh-aGan-aGan } & \text { na } & \text { l-wosh-aGan-aGan-ek } \\ \text { 3T-cocinar-ANTIPAS-CAUS } & \text { DAC } & \text { 3POS-cocinar-ANTIPAS-CAUS-RES(=MASC) }\end{array}$

'(él) hace cocinar a alguien' 'este su cocinero'

lit.: 'su hombre al que ordena cocinar'

a-na l-wosh-aGan-aGan-a
fem-dac $\quad 3$ pos-cocinar-ANTIPAS-CAUS-PAC(=FEM)
'esta su cocinera'
lit.: 'su mujer a la que ordena cocinar'

j-era-Gan-aGan

3T-escribir-CAUS-CAUS

na 1-era-aGan-aGan-ek

'(él) hace escribir (a alguien)'

DAC 3POS-escribir-ANTIPAS-CAUS-RES(=MASC)

'este su secretario'
a-na 1-era-aGan-aGan-a
DAC-FEM 3POS-escribir-ANTIPAS-CAUS-PAC(=FEM)
'esta su secretaria'

En otros nombres derivados con el sufijo - $a$ se puede observar que la alternancia entre $-\varnothing \mathrm{y}-k$ como clasificadores de forma posee un correlato de asignación de género femenino y masculino respectivamente: 
(30) i-wet

$3 \mathrm{~T}$-tener dolor

'(él) tiene dolor en' [TRN] 1-wet-a- $\varnothing$

3POS-tener dolor-PAC-FEM

'este su grano' [FEM]

na l-wet-a-k

DAC 3POS-tener dolor-PAC-MASC

'esta su lastimadura' [MASC]

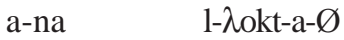

FEM-DAC 3POS-remar-PAC-FEM

'esta su canoa' [FEM]

na $\quad 1-\lambda$ okt-a-k

DAC 3POS-remar-PAC-MASC

'este su flotante' [MASC]

a-na 1-asowaqt-a- $\varnothing$

FEM-DAC 3POS-perjudicar-PAC-FEM

'esta su perjudicada' [FEM]

na 1-asowaqt-a-k

DAC 3POS-perjudicar-PAC-MASC

'este su perjudicado' [MASC]

a-na 1-apaGagen-ata- $\varnothing$

FEM-DAC 3POS-ens./apr.-ANTICAU/PAC-FEM

'esta su alumna' [FEM]

na 1-apaGagen-ata-k

DAC 3POS-ens./apr.-ANTICAU/PAC-MASC

'este su alumno' [MASC]

Los ejemplos (30) a (33) podrían estar indicando el surgimiento de una nueva regla de asignación fonológica de género: los nombres derivados que finalizan con el fonema $-k$ indican género natural masculino. Esta regla se aplica a un porcentaje altísimo de nombres, salvo que contradigan reglas de asignación semántica, como la que rige los nombres de árboles y otros vegetales: ara mapik 'este algarrobo' [FEM]. A su vez, otro sufijo nominalizador agentivo -naq / -aq, que por regla de asignación morfológica selecciona el género masculino, admite un morfema - $a$ para marcar género femenino, procedimiento que se podría interpretar como otro indicio del reanálisis del morfema pacientivo de los ejemplos (29) a (33) en indicador de género natural femenino: 
(34)

ra-pjoGon

3IA-lamer

'(él) lame' [INTR]

$$
\begin{aligned}
& \text { na } \quad \text { pjoGon-aq- } \varnothing \\
& \text { DAC lamer-P2-MASC } \\
& \text { 'este chamán' }[\text { MASC] } \\
& \\
& \begin{array}{ll}
\text { a-na } & \text { pjoGon-aG-a } \\
\text { FEM-DAC } & \text { lamer-P2-FEM } \\
\text { 'esta chamana' [FEM] }
\end{array}
\end{aligned}
$$

(35)

PoleGra 'gallo / gallina'

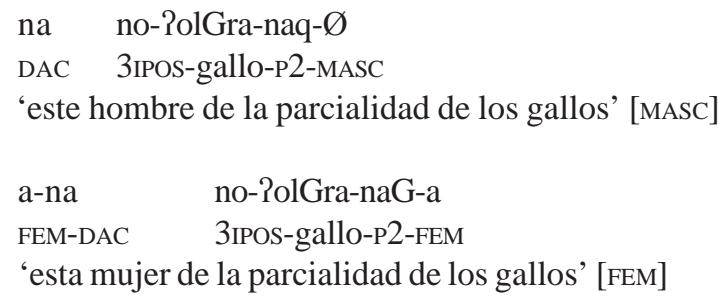

Si bien la tendencia a reutilizar el sufijo nominalizador pacientivo como indicador de género natural femenino parece extenderse, todavía está muy lejos de conformar una regla de asignación fonológica, ya que muchísimos nombres no derivados finalizados en /-a\#/ seleccionan el género masculino (na alaGa 'esta venda', na laita 'este su olor', etc.).

\subsection{Un único afijo bigenérico: el clasificador $-k i$}

El clasificador LUGAR CERRADO, CON LÍMITES DEFINIDOS / RECIPIENTE es el único sufijo nominalizador que puede seleccionar cualquiera de los dos géneros siguiendo, aparentemente, un criterio semántico: los lugares u objetos grandes seleccionan el género masculino y los pequeños el femenino:

(36) i-men

3T-vender

'(él/ella) vende'

r-an-aGan

3IA-dar-ANTIPAS

'(él/ella) siembra'

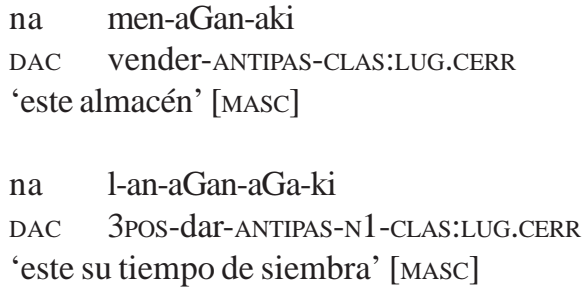

(37)

a-na ltaGaki 'esta su olla' [FEM] na lalojaGaki 'este corral' [MASC] a-na l-apjaGaki ‘este su estribo’ [FEM] na l-aponaGaki 'esta casa de reunión’ [MASC] a-na l-ashiiraGanaGaki 'esta su pipa' [FEM] na l-woshaGaki ‘esta su cocina' [MASC] a-na l-ko?oGoki 'este su útero' [FEM] na l-awoGonaGaki 'este su horno’ [MASC] a-na 1-chaGaki 'esta su vejiga' [FEM] 
Sin embargo, esta regla no se verifica en los ejemplos (38), posiblemente por tratarse de préstamos del español:
(38) a-na l-atannaGanaGaki
'este su hospital donde cura' [FEM]
a-na l-eraGanaGaki
a-na r-asotaGaki
'esta su biblioteca'; 'esta su escuela' [FEM]
a-na l-PonaGanaGaki
'esta su pista de baile' [FEM]
'esta su casa de canción'; 'esta su iglesia' [FEM]

\subsection{Distinción de homófonos y otras utilizaciones}

Las diferencias de género permiten distinguir los homófonos:

\begin{tabular}{|c|c|c|}
\hline 1-chi & a-na & 1-chi \\
\hline 3Pos-espuma & FEM-DAC & 3POS-pierna \\
\hline 'esta su espuma' [MASC] & 'esta su p & $\mathrm{a}^{\prime}$ [FEM] \\
\hline
\end{tabular}

wo?o ra 1-wik a-ñi l-wik

PRES.EX DPA 3POS-necesidad FEM-DSE 3POS-punta de flecha de carandá

lit.: 'hay esta su necesidad' [MASC] 'esta su punta de flecha de carandá' [FEM]

$\begin{array}{lllc}3 \mathrm{i} & \text { rapichi? } & \text { a-na } & \text { rapichi? } \\ \text { DACOS } & \text { const. Las Pléyades } & \text { FEM-DAC } & \text { clavel del aire } \\ \text { 'este Rapichi?' }[\mathrm{MASC}]^{8} & \text { 'este clavel del aire' [FEM] }\end{array}$

En muy pocos casos, la marca de género de un nombre puede cambiar para explotar al máximo los recursos semánticos de la lengua, como el ejemplo encontrado en el Vocabulario Toba de Buckwalter (1980):
(40) na ala?
$\begin{array}{ll}\text { a-na } & \text { ala? } \\ \text { FEM-DAC } & \text { fruto }\end{array}$
'esta fruta alargada' [MASC]
'esta fruta redonda' [FEM]

\subsection{Préstamos}

Todavía no hemos identificado la regla de asignación de género de los préstamos en los textos libres estudiados. En muchos casos se respeta el género de la lengua dadora:
na nacimiento
re?era evangelio
'este evangelio' [MASC]
so lote 'este nacimiento' [MASC]
ra seguro
'este lote' [MASC]
'este seguro' [MASC]

${ }^{8}$ Para los tobas, Rapichi 2 es un hombre anciano y la constelación se parece a la planta parásita comúnmente denominada 'clave del aire’ (Gómez, 2007). 
en otros el de la lengua toba:

(42) ana?ana libro 'este libro' [FEM] ana lere 'este su libro' [FEM]

Es habitual la selección del género femenino para objetos pequeños o redondos, o cuando el nombre finaliza en vocal/_e\#/(regla de asignación fonológica):

$$
\begin{array}{ll}
\text { aye karné } & \text { 'este carnet' [FEM] } \\
\text { ana bala } & \text { 'esta bala' [FEM] } \\
\text { ana?ana alambre } & \text { 'este alambre' [FEM] }
\end{array}
$$

\begin{tabular}{|c|c|c|}
\hline \multirow[t]{5}{*}{ (44) } & so tierra & 'esta tierra' [MASC] \\
\hline & na waka & 'esta vaca' [MASC] \\
\hline & ra costumbre & 'esta costumbre' [MASC] \\
\hline & na lista & 'esta lista' [MASC] \\
\hline & ra la historia & 'esta historia' [MASC] \\
\hline
\end{tabular}

mientras que la regla de asignación basada en la forma o tamaño (objetos grandes, también nombres abstractos como masculinos) parece operar en préstamos tales como:

\section{CONCLUSIONES}

En este trabajo presentamos las reglas de asignación semántica, fonológica y morfológica del género natural y gramatical en toba. En la mayoría de los nombres los criterios de asignación no presentan excepciones, salvo para los derivados con el morfema locativo - $k i$, cuyos criterios de asignación no hemos logrado dilucidar. También presentamos nombres derivados donde pudimos observar procesos de reanálisis del significado de sus morfemas derivativos; en algunos casos fue posible observar la superposición o coalescencia de los criterios (clasificatorio vs. genérico) lo que nos permite establecer una posible ruta de gramaticalización entre la noción de tamaño pequeño y/o forma redonda con la de género femenino, y entre tamaño grande y/o forma alargada con el género masculino. Otros dos temas restan ser estudiados en relación a las clases nominales o géneros: en primer lugar su interrelación con la clase del número y su expresión en el sistema de alineación de los argumentos nominales; en segundo lugar un estudio de la frecuencia de utilización de uno u otro género gramatical en distintos géneros de habla, investigación que puede echar luz sobre los procesos de gramaticalización en curso, así como de fenómenos de variación debido a diferencias diatópicas y cronolectales.

\section{BIBLIOGRAFÍA}

AIKHENVALD, A. (2000). Classifiers. A Typology of Noun Categorization Devices. Oxford / New York: Oxford University Press. 
BUCKWALTER, A. (1980). Vocabulario Toba - Seguido de algunos apuntes sobre la gramática del idioma toba. Roque Saénz Peña Chaco (Argentina): edición del autor.

CARPIO, M. B. (2004). La categoría de número en la lengua toba (familia Guaycurú, Argentina). Tesis de Licenciatura en Antropología. Universidad Nacional de Rosario, ms.

CENSABELLA, M. (1997). Dynamique phonologique de la langue toba. La Linguistique, Paris: P.U.F., Vol. 33/1: 95-109.

.(2002). Descripción funcional de un corpus en lengua toba (familia Guaycurú, Argentina). Sistema fonológico, clases sintácticas y derivación. Aspectos de sincronía dinámica, ms. presentado para la obtención del doctorado en Letras Modernas de la Universidad Nacional de Córdoba, 324p. $\mathrm{y}$ anexos.

.(2006). 'Relaciones gramaticales en la lengua toba (familia. Guaycurú, Argentina)' en Estrada Fernández, Z. (ed.) Actas del VIII Encuentro Internacional de Lingüística en el Noroeste, pp. 81103. División de Humanidades y Bellas Artes, Dpto. de Letras y Lingüística, Universidad de Sonora, México.

CORBETT, G. (1991). Gender. Cambridge: Cambridge University Press.

GOMEZ, C. (2007). Algunas representaciones Toba-Pilagá sobre las constelaciones celestes. Actas XVI Encuentro de Geohistoria Regional, Resistencia: Instituto de Investigaciones Geohistóricas.

GRONDONA, V. (1998). A grammar of mocovi. Ph.D. Thesis. University of Pittsburg.

GUALDIERI, C. B. (1998). Mocovi (Guaicuru). Fonologia e morfossintaxe. Tese de Doutorado. Universidade Estadual de Campinas.

.(2007). Clasificadores guaycurúes: un desafío para la lingüística. Actas $1^{\circ}$ Encuentro de Lenguas Indígenas Americanas. Santa Rosa: Universidad Nacional de La Pampa.

INDEC. (2007). Resultados de la Encuesta Complementaria de Pueblos Indígenas (ECPI) 2004-2005. Disponible en http://www.indec.mecon.gov.ar/webcenso/ECPI/index_ecpi.asp

KIRTCHUK, P. (1996). Lingüística areal: Deixis y clasificación nominal del Gran Chaco. En: H. Martín y J. Pérez Diez (comp.) Lenguas indígenas de Argentina, pp. 75-85 San Juan: Ed. Fundación Universidad Nacional de San Juan.

KLEIN, H. M. (1979). Noun classifiers in Toba. En: M. Mathiot (ed.). Ethnolinguistics: Boas, Sapir and Whorf revisited pp. 85-95. The Hague: Mouton.

MARTINET, A. (1985). Syntaxe Générale, París: Armand Colin.

MESSINEO, C. (1991). Variantes dialectales del complejo lingüístico toba. En J. Braunstein, (ed.) Hacia una nueva carta étnica del Chaco, $\mathrm{N}^{\circ} \mathrm{II}, \mathrm{pp} .13-22$. Las Lomitas, Formosa: Centro CHACO. .(1998). El valor estilístico de las partículas deícticas en la lengua Toba. Actas III Jornadas de Lingüística Aborigen, pp. 443-456. Buenos Aires: Instituto de Lingüística, F.F. y L., UBA.

(2002). El contraste alienable / inalienable en las construcciones posesivas del toba (guaycurú). En Temas de Lingüística Aborigen, Vol 2: 53-81, Archivo de Lenguas Indoamericanas, F. F. y L., Universidad de Buenos Aires.

SANDALO, F. (1995). A Grammar of Kadiwéu. Ph.D. Thesis. University of Pittsburg.

TRUDGILL, P. (2002). Sociolinguistic Variation and Change, Washington DC: Georgetown University Press. 
Censabella: Critérios de asignación de GÉnERo gramatical en toba

SANDALO, F. (1995). A Grammar of Kadiwéu. Ph.D. Thesis. University of Pittsburg.

TRUDGILL, P. (2002). Sociolinguistic Variation and Change, Washington DC: Georgetown University Press.

VIDAL, A. (1997). Noun classificación in Pilagá (Guaykuruan). The Journal of Amazonian Languages, Vol. I: 58-111.

WILBERT, J. \& K. SIMONEAU. (1982). Folk Literature of the Toba Indians. Los Angeles: University of California.

\section{Abreviaturas}

\begin{tabular}{|c|c|}
\hline $1,2,3$ & 1ra, 2da, 3ra persona \\
\hline AB.HORIZ & "abajo. horizontal» \\
\hline $\mathrm{AD}$ & «adentro» \\
\hline ADES & agente desconocido \\
\hline ANTICAUS & anticausativo \\
\hline ANTIPAS & antipasiva \\
\hline APL & aplicativo \\
\hline CAU & causativo \\
\hline CLAS & clasificador \\
\hline DAC & determinante demostrativo "acercamiento" \\
\hline DAL & determinante demostrativo "alejamiento" \\
\hline DNP & determinante demostrativo "no presente" \\
\hline DPA & determinante demostrativo "parado" \\
\hline DSE & determinante demostrativo "sentado" \\
\hline FEM & femenino \\
\hline GENT & sufijo "gentilicio" \\
\hline IA & evento intransitivo agentivo \\
\hline INSTR, 1 & sufijos "instrumentales" \\
\hline LUG & lugar \\
\hline LUG.CERR & "lugar. cerrado" \\
\hline M & voz media \\
\hline N1 & sufijo nomlzdor. "nombre de acción" \\
\hline $\mathrm{N} 2$ & sufijo nomlzdor. "nombre de acción voluntaria" \\
\hline MASC & masculino \\
\hline $\mathrm{P}$ & pronombre personal \\
\hline $\mathrm{P} 1,2,3,4$ & sufijos derivativos "agente de la acción" \\
\hline PAC & sufijo "paciente de la acción" \\
\hline PDAC & pronombre demostrativo "acercamiento" \\
\hline PDAC & pronombre demostrativo "acostado" \\
\hline PDSE & pronombre demostrativo "sentado" \\
\hline
\end{tabular}


PL

plural

POS

PRES.DEM.AC

PROG

RES

$\mathrm{T}$

posesivo

presentativo demostrativo "acercamiento"

aspecto verbal "progresivo"

sufijo "resultado de la acción"

evento transitivo

VERB

sufijo "verbalizador"

Recebido 05/02/2007

Versão Corrigida: 10/10/2007

Aceito: 04/03/2008 